\title{
Successful thrombolytic therapy with recombinant tissue- type plasminogen activator for massive pulmonary embolism -A case report-
}

\author{
Min Soo Kim, Kye-Min Kim, Seung-Hoon Woo, Yun Hee Lim, Jun Heum Yon, and Seung-Gyu Jeon \\ Department of Anesthesiology and Pain Medicine, Sanggye Paik Hospital, Inje University College of Medicine, Seoul, Korea
}

Massive pulmonary embolism is associated with significant perioperative morbidity and mortality. We report here on a case of a 69-year-old man who suffered a massive pulmonary embolism with pulseless electrical activity during knee arthroscopic surgery. After a diagnosis was made by performing transthoracic echocardiography, the patient was treated with recombinant tissue-type plasminogen activator. The patient was transferred to the intensive care unit after his hemodynamic status improved. The patient went on to make a full cardiopulmonary recovery without any complications. (Korean J Anesthesiol 2010; 59: 56-60)

Key Words: Pulmonary embolism, Thrombolytic therapy, Tissue plasminogen activator.

Intraoperative acute pulmonary thromboembolism is a devastating condition that has a very high risk of mortality, and it requires prompt diagnosis and treatment. However, this condition is difficult to diagnose when the patient is under general anesthesia due to the nonspecific clinical findings and the lack of signs. Treatment should be started immediately since severe pulmonary embolism leads to rapid hemodynamic deterioration. Although thrombolysis is widely accepted as the front-line therapy for most patients with massive pulmonary embolism, the treatment fails in $15-20 \%$ of patients [1]. Surgical embolectomy has been reserved for the severely compromised patients or for those in whom thrombolytic therapy has failed.
However, the mortality after surgical embolectomy remains at approximately $27 \%$ [2].

Here we report on a case of massive pulmonary embolism that occurred during general anesthesia, and the patient was successfully treated with alteplase, a recombinant tissue-type plasminogen activator (r-tPA).

\section{Case Report}

A 69-year-old man (height, $170 \mathrm{~cm}$; weight, $80 \mathrm{~kg}$ ) was scheduled for repeated arthroscopic lavage of the right knee for the treatment of unimproved septic arthritis in spite of

Received: December 28, 2009. Revised: January 21, 2010. Accepted: February 5, 2010.

Corresponding author: Kye-Min Kim, M.D., Ph.D. Department of Anesthesiology and Pain Medicine, Sanggye Paik Hospital, Inje University College of Medicine, 761-1, Sanggye 7-dong, Nowon-gu, Seoul 139-707, Korea. Tel: 82-2-950-1173, Fax: 82-2-950-1323, E-mail: kyemin@paik.ac.kr This case was presented at 86th Congress of Korean Society of Anesthesiologists. November 7th, 2009. Jeju International Convention Center. (c) This is an open-access article distributed under the terms of the Creative Commons Attribution Non-Commercial License (http:// creativecommons.org/licenses/by-nc/3.0/), which permits unrestricted non-commercial use, distribution, and reproduction in any medium, provided the original work is properly cited. 
the previous procedure that had been carried out 2 weeks previously. At the evaluation done 2 weeks ago, it was found that he had peripheral arterial occlusive disease in the lower extremities, but there was no evidence of deep vein thrombosis. He was being treated for hypertension and type 2 diabetes. During the first arthroscopic lavage under general anesthesia, new onset atrial fibrillation with a rapid ventricular response was noted. The transthoracic echocardiography done at that time showed left atrial enlargement, normal left ventricular function with an ejection fraction of $61.3 \%$ and there was no evidence of intracardiac thrombus. Flecainide and aspirin were prescribed for the atrial fibrillation.

Before the second operation, the preoperative electrocardiogram showed normal sinus rhythm. Intramuscular injection of glycopyrrolate $0.2 \mathrm{mg}$ was given as premedication. Anesthesia was induced with propofol and rocuronium and the airway was secured with a cuffed $7.5 \mathrm{~mm}$ endotracheal tube. The electrocardiogram, pulse oxygen saturation, capnogram and esophageal temperature were monitored. The invasive arterial pressure was also monitored because the vital signs were unstable during the previous operation due to atrial fibrillation and the septic condition. Anesthesia was maintained with sevoflurane and $50 \%$ nitrous oxide in oxygen. Arterial blood gas analysis that was done 10 minutes after induction of anesthesia showed $\mathrm{pH}$ 7.38, $\mathrm{PaCO}_{2} 45.1 \mathrm{mmHg}, \mathrm{PaO}_{2} 85.7 \mathrm{mmHg}, \mathrm{HCO}_{3}{ }^{-}$ $26.0 \mathrm{mEq} / \mathrm{L}$ and $\mathrm{SaO}_{2} 95.5 \%\left(\mathrm{FiO}_{2}: 0.5\right)$. The end tidal $\mathrm{CO}_{2}$ was 24 $\mathrm{mmHg}$. The tidal volume was increased to $750 \mathrm{ml}$ from $650 \mathrm{ml}$. Twenty minutes after changing the ventilator setting, arterial blood gas analysis showed $\mathrm{pH}$ 7.31, $\mathrm{PaCO}_{2} 47.0 \mathrm{mmHg}, \mathrm{PaO}_{2}$ $75.1 \mathrm{mmHg}, \mathrm{HCO}_{3}{ }^{-} 23.6 \mathrm{mEq} / \mathrm{L}$ and $\mathrm{SaO}_{2} 92.8 \%\left(\mathrm{FiO}_{2}\right.$ : 0.5).

Phenylephrine $100 \mu \mathrm{g}$ was injected intravenously at 30 minutes after induction because the blood pressure started to drop to $90 / 60 \mathrm{mmHg}$ and the heart rate increased to 100 beats per minute. Forty minutes after anesthetic induction when the surgical procedure was almost completed, the arterial blood pressure dropped to $65 / 45 \mathrm{mmHg}$ with a heart rate of 48 beats per minute. Epinephrine $30 \mu \mathrm{g}$ and then an additional 50 $\mu \mathrm{g}$ of peinephrine and atropine sulfate $0.5 \mathrm{mg}$ were injected intravenously. However, the vital signs deteriorated and the pulsatile activity disappeared in the arterial line. Cardiopulmonary resuscitation (CPR) was immediately started and the surgery was completed. The nitrous oxide and sevoflurane were turned off. Epinephrine $1 \mathrm{mg}$ and atropine sulfate 0.5 mg were administered intravenously. The vital signs were not improved in spite of intermittent administration of epinephrine. The arterial blood gas analysis showed $\mathrm{pH}$ 7.37, $\mathrm{PaCO}_{2} 27.8$ $\mathrm{mmHg}, \mathrm{PaO}_{2} 63.9 \mathrm{mmHg}, \mathrm{HCO}_{3}{ }^{-} 15.8 \mathrm{mEq} / \mathrm{L}$ and $\mathrm{SaO}_{2} 90.8 \%$ $\left(\mathrm{FiO}_{2}: 1.0\right)$.

Eight minutes after starting CPR, external cardiac massage was stopped because the vital signs were restored. Arterial blood gas analysis showed $\mathrm{pH}$ 6.96, $\mathrm{PaCO}_{2} 63.9 \mathrm{mmHg}, \mathrm{PaO}_{2} 221.4 \mathrm{mmHg}$, $\mathrm{HCO}_{3}{ }^{-} 13,9 \mathrm{mEq} / \mathrm{L}$ and $\mathrm{SaO}_{2} 98.5 \%\left(\mathrm{FiO}_{2}: 1.0\right)$. Thereafter, with the vital signs fluctuating severely, the CPR was intermittently continued with the administration of epinephrine and sodium bicarbonate on the basis of the hemodynamic status and the results of the arterial blood gas analysis. Ten minutes after initiation of CPR, an emergency transthoracic echocardiogram was performed with the impression of pulmonary embolism and it showed a massive amount of thrombus in the right atrium and a dilated hypokinetic right ventricle with a D shaped left ventricle (Fig. 1). Under the preliminary diagnosis of massive pulmonary embolism, the decision was made to proceed with fibrinolysis while the CPR was ongoing. Thirty-seven minutes after the initiation of CPR, $20 \mathrm{mg}$ of alteplase, which is a r-tPA (Actylase $^{\circledR}$, Boehringer Ingelheim, Ingelheim, Germany), was administered intravenously and $100 \mathrm{mg}$ was continuously infused over 90 minutes. The echocardiogram after injection of r-tPA showed improvement of the D shaped left ventricle, but multiple, mobile thrombi in the right atrium were still present (Fig. 2). The hemodynamic performance of the systemic and
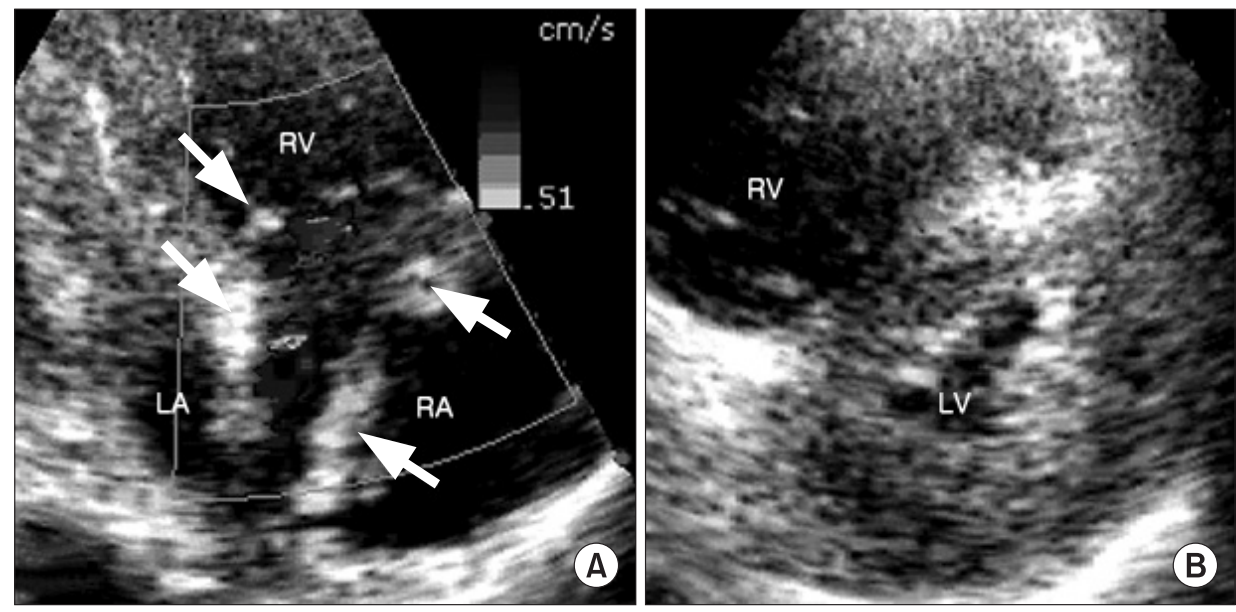

Fig. 1. (A) Transthoracic echocardiography shows multiple, mobile hyperechoic thrombi (arrows) in the right atrium (RA) and marked dilatation of the RA and right ventricle (RV). (B) The shifted interventricular septum to the left ventricular (LV) side (D-shaped left ventricle) during diastole, as seen on the parasternal short axis view. LA: left atrium. 

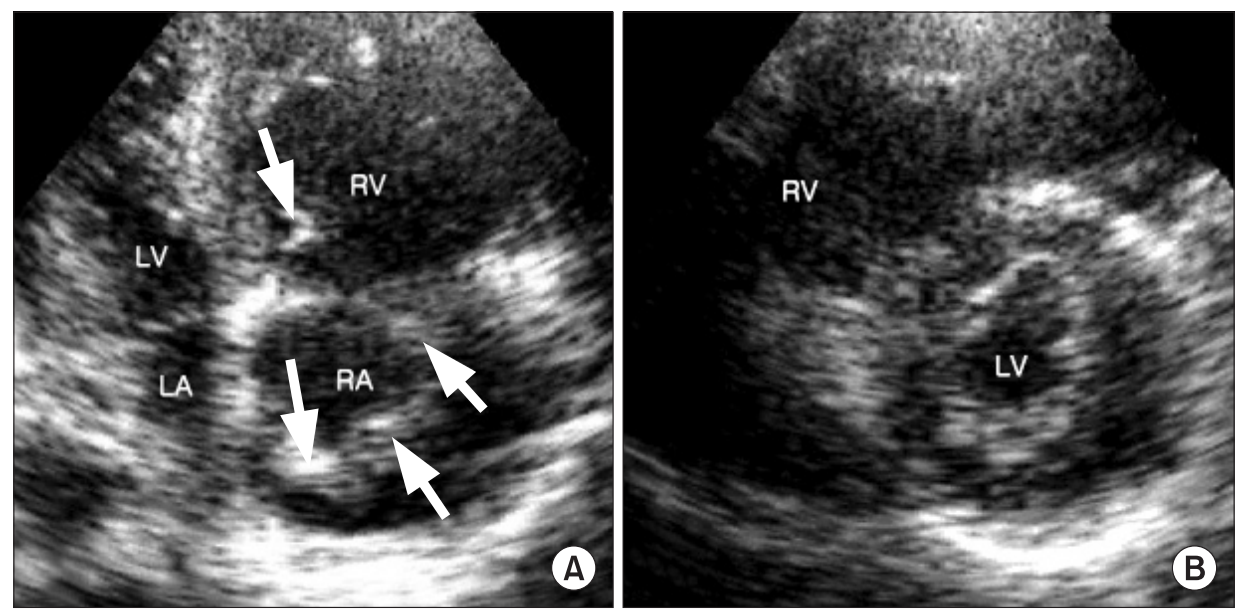

Fig. 2. (A) The remaining multiple, mobile thrombi (arrows) in the right atrium (RA) after thrombolysis, as seen on the apical four chamber view. (B) Improvement of the D-shaped left ventricle (LV) after thrombolysis, as seen on the parasternal short axis view. RV: right ventricle, LA: left atrium.
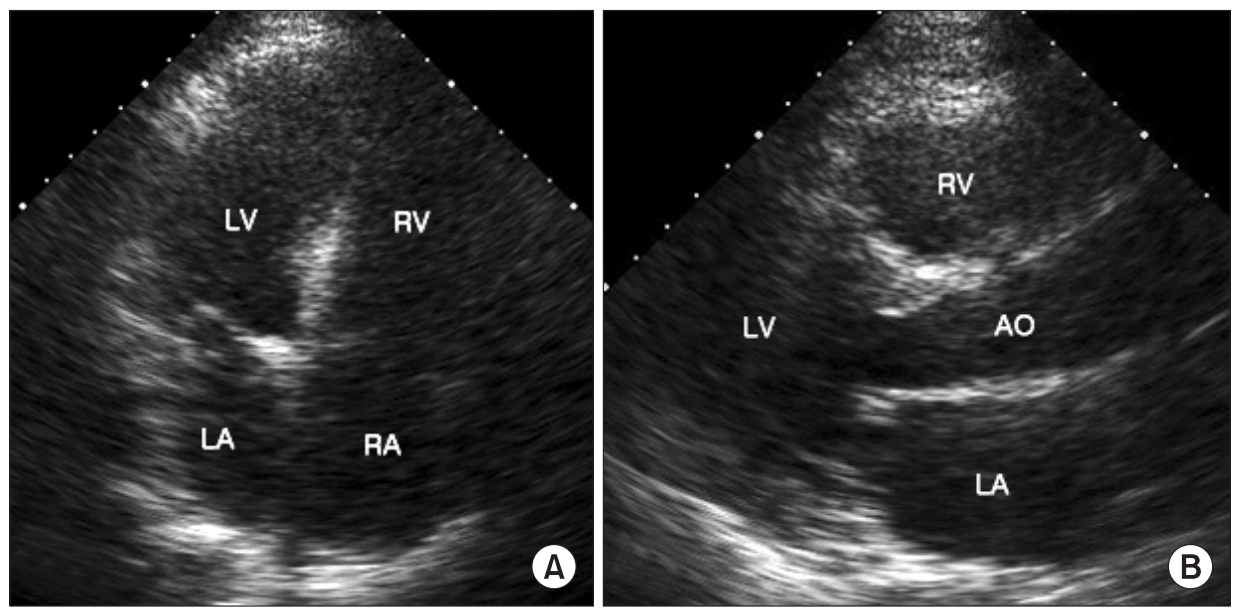

Fig. 3. (A) The follow-up transthoracic echocardiography after 3 days. There are no visible intracardiac thrombi seen on the apical four chamber view. (B) The sizes of the ventricles are normalized, as seen on the parasternal long axis view. RA: right atrium, RV: right ventricle, LA: left atrium, LV: left ventricle, $\mathrm{AO}$ : aorta.

pulmonary circulation was stabilized. There was no sign of bleeding at the operation site. The external cardiac massage was stopped 26 minutes after injecting the recombinant tissue-type plasminogen activator. The patient, who was in an intubated state, was transferred to the intensive care unit. As the patient was transferred to the intensive care unit, the blood pressure increased to $110 / 60 \mathrm{mmHg}$ and the heart rate was 100 beats per minute, with mechanical ventilation. Heparin was given 12 hours after injecting the r-tPA. The PT was INR was 1.65 and the aPTT was 50-70 seconds. Eighteen hours later, the patient's consciousness became alert and the endotracheal tube was extubated.

The thrombi had completely disappeared and the dilated hypokinetic right ventricle had returned to normal, as was noted on the echocardiogram performed 3 days after surgery (Fig. 3). The heparin infusion was stopped 5 days after surgery and patient recovered completely without any hemorrhagic episodes. He was transferred from the intensive care unit to the medical ward.

\section{Discussion}

The representative therapeutic modalities for treating pulmonary embolism include medical treatment with thrombolytics and surgical measures. Although massive pulmonary embolism that causes hemodynamic instability has generally been treated with surgical embolectomy, it seems that fibrinolytic agents are now being increasingly advocated as the first line therapy for such a condition [3].

As the causes of perioperative pulmonary embolism, 80 to $90 \%$ of it originates from deep vein thrombosis and only $71 \%$ of these patients can survive over 1 week [4]. Prolonged immobility, obesity and an age more than 60 years are considered to be additional risk factors for pulmonary thromboembolism. Even though there was no evidence of deep vein thrombosis 2 weeks before surgery, such risk factors for pulmonary thromboembolism as an older age, 2 weeks of immobilization and obesity were all present in this current case. Therefore, we suspected deep vein thrombosis to be the cause of the patient's 
pulmonary thromboembolism.

Cyanosis, hypoxemia, hypotension, pulmonary hypertension and an increased central venous pressure are signs of pulmonary embolism during general anesthesia [4]. Wheezing in the breathing sounds can be heard and decreased lung compliance is observed. Nonspecific findings such as sudden hypocapnia with hypotension may be a sign of pulmonary embolism. An echocardiogram can be performed in an emergency situation for making the diagnosis of pulmonary embolism. This is useful to monitor the intracardiac structures and the size, shape and motility of the intracardiac thrombi. The presence of pulmonary thromboembolism can be highly suspected when observing dilatation of the right atrium and right ventricle, a shifted interventricular septum to the left ventricular side (D-shaped left ventricle) and pulmonary hypertension [5]. Echocardiography has emerged not only as a diagnostic tool for the detection of right heart thrombi associated with pulmonary embolism, but also as an aid for monitoring the effects of treatment and detecting possible complications [5].

The treatment of pulmonary thromboembolism is still controversial. In this case, the massive pulmonary embolism that caused pulseless electrical activity was successfully treated with thrombolytic therapy. Catastrophic bleeding can occur with thrombolytic therapy. In a series of 312 patients who received thrombolytic therapy for pulmonary embolism, there was a $1.9 \%$ incidence of intracranial bleeding [6]. In another study including 132 consecutive patients received alteplase for massive pulmonary embolism, both intracranial bleeding and pericardial tamponade occurred with an incidence of 1.5\% [7]. Other major bleeding complications included gastrointestinal hemorrhage, hemoptysis and hematomas at the puncture site for pulmonary angiography. Autopsy studies have shown that hemorrhagic complications can be estimated to occur in more than $15 \%$ of all patients who are undergoing thrombolysis after CPR [7]. Despite complications such as intracranial hemorrhage, thrombolysis remains the treatment of choice for the majority of these patients. Our patient was fortunate enough to make a full neurological and cardiopulmonary recovery without any complications. A review of 34 patients who were given thrombolytic agents during CPR for massive pulmonary embolism suggested an initial survival rate of 55$100 \%$ [7]. By far, the most common thrombolytic drugs that have been used to thrombolyse pulmonary embolism are urokinase, streptokinase and alteplase [8]. Alteplase is currently the recommended drug because streptokinase may exacerbate hypotension, while the dose for urokinase treatment is too low for use in patients with pulmonary embolism [9]. The patients treated with alteplase infusions had a significantly lower mortality rate and significantly fewer major bleeding episodes than did the patients who were treated with streptokinase [9].
Once bound to fibrin clots, alteplase has a short duration of action, with a plasma half-life of $4-5$ minutes. The fibrinolytic therapy action of alteplase will continue at the site of fibrinbound plasminogen [10]. The recommended dose of alteplase is $100 \mathrm{mg}$ as a peripheral IV infusion over 2 hours, although considerable variation in the dosing has reported by several investigators [11].

Apart from thrombolysis, surgical intervention and the combination of mechanical catheter fragmentation and subsequent lysis represent specific therapeutic options during CPR. However, they may only be effective for treating central pulmonary embolism. Acute surgical embolectomy during CPR is only an alternative in those hospitals where the patient can be placed on an extracorporeal bypass system. This approach is usually successful if it is undertaken in patients with stable hemodynamics [12]. Surgical embolectomy is associated with a maximal survival rate of 20 to $55 \%$ during CPR [13,14]. These techniques also may be an alternative after unsuccessful thrombolytic treatment and CPR [14].

Our case had an episode of massive pulmonary embolism and this was accompanied by cardiac arrest that required CPR. The prognosis of patients with life-threatening pulmonary embolism can be significantly improved with an early diagnosis and starting treatment without delay [4]. In our opinion, we should have detected the pulmonary embolism earlier so that thrombolysis could have been initiated at an earlier time. A high clinical suspicion of impending pulmonary thromboembolism, according to the signs of hypoxemia, sudden hypocapnia and hypotension, is mandatory for diagnosing the susceptible patients who have risk factors for pulmonary embolism, such as prolonged immobility, obesity and old age, and these risk factors were already present in our patient. Early initiation of thrombolytic therapy is essential and this should be done in any patient with pulmonary thromboembolism, except if there is an absolute contraindication. This case provides further evidence that thrombolytic therapy may be beneficial for patients with pulmonary embolism that has induced cardiac arrest.

\section{References}

1. Tayama E, Ouchida M, Teshima H, Takaseya T, Hiratsuka R, Akasu $\mathrm{K}$, et al. Treatment of acute massive/submassive pulmonary embolism. Circ J 2002; 66: 479-83.

2. Dauphine C, Omari B. Pulmonary embolectomy for acute massive pulmonary embolism. Ann Thorac Surg 2005; 79: 1240-4.

3. Wood KE. Major pulmonary embolism: review of a pathophysiologic approach to the golden hour of hemodynamically significant pulmonary embolism. Chest 2002; 121: 877-905.

4. Farfel Z, Shechter M, Vered Z, Rath S, Goor D, Gafni J. Review of echocardiographically diagnosed right heart entrapment of pulmonary emboli-in-transit with emphasis on management. Am 
Heart J 1987; 113: 171-8.

5. Kasper W, Meinertz T, Kersting F, Löllgen H, Limbourg P, Just H. Echocardiography in assessing acute pulmonary hypertension due to pulmonary embolism. Am J Cardiol 1980; 45: 567-72.

6. Kanter DS, Mikkola KM, Patel SR, Parker JA, Goldhaber SZ. Thrombolytic therapy for pulmonary embolism. Frequency of intracranial hemorrhage and associated risk factors. Chest 1997; 111: 1241-5.

7. Spöhr F, Böttiger BW. Safety of thrombolysis during cardiopulmonary resuscitation. Drug Saf 2003; 26: 367-79.

8. Böttiger BW, Böhrer H, Bach A, Motsch J, Martin E. Bolus injection of thrombolytic agents during cardiopulmonary resuscitation for massive pulmonary embolism. Resuscitation 1994; 28: 45-54.

9. Semba CP, Bakal CW, Calis KA, Grubbs GE, Hunter DW, Matalon $\mathrm{TA}$, et al. Alteplase as an alternative to urokinase. Advisory Panel on Catheter-Directed Thrombolytic Therapy. J Vasc Interv Radiol 2000;
11: 279-87.

10. Capstick T, Henry MT. Efficacy of thrombolytic agents in the treatment of pulmonary embolism. Eur Respir J 2005; 26: 864-74.

11. British Thoracic Society Standards of Care Committee Pulmonary Embolism Guideline Development Group. British Thoracic Society guidelines for the management of suspected acute pulmonary embolism. Thorax 2003; 58: 470-83.

12. Meyer G, Gisselbrecht M, Diehl JL, Journois D, Sors H. Incidence and predictors of major hemorrhagic complications from thrombolytic therapy in patients with massive pulmonary embolism. Am J Med 1998; 105: 472-7.

13. Ullmann M, Hemmer W, Hannekum A. The urgent pulmonary embolectomy: mechanical resuscitation in the operating theatre determines the outcome. Thorac Cardiovasc Surg 1999; 47: 5-8.

14. Clarke DB, Abrams LD. Pulmonary embolectomy: a 25 year experience. J Thorac Cardiovasc Surg 1986; 92: 442-5. 